\title{
Funktionelle Stuhlinkontinenz bei Kindern
}

\author{
Marloes E.J. Bongers Marc A. Benninga \\ Department of Pediatric Gastroenterology and Nutrition, Emma Children's Hospital, Academic Medical Center, \\ Amsterdam, The Netherlands
}

\author{
Schlüsselwörter \\ Funktionelle Stuhlinkontinenz • Enkopresis • Obstipation \\ im Kindesalter
}

\section{Zusammenfassung}

Stuhlinkontinenz bezeichnet die unwillkürliche Stuhlentleerung. Dieses frustierende Symptom verursacht Kindern und ihren Familien erhebliches Leid und beträchtliche Belastungen. Die Stuhlinkontinenz im Kindesalter lässt sich in zwei Hauptkategorien unterteilen: (1) die organische Stuhlinkontinenz, die sich z. B. infolge anorektaler Missbildungen oder neurogener Störungen ergibt, und (2) die nichtorganische bzw. funktionelle Stuhlinkontinenz. Dieser Übersichtsartikel konzentriert sich ausschliesslich auf die funktionelle Stuhlinkontinenz bei Kindern und geht auf Definition, Epidemiologie, Pathophysiologie und die neueren Erkenntnisse hinsichtlich klinischer und diagnostischer Aspekte ein.

Copyright $\odot 2008$ Nestec Ltd., Vevey/S. Karger AG, Basel

\section{Definition}

Bisher wurden die Begriffe Enkopresis und Stuhlschmieren (Soiling) beliebig austauschbar zur Beschreibung der nichtorganischen (funktionellen) Stuhlinkontinenz verwendet. Inzwischen unterscheiden viele Pfleger diese beiden Begriffe nach der ausgeschiedenen Stuhlmenge [1]. Während Enkopresis wie folgt definiert wird: Wiederholtes willkürliches oder unwillkürliches Abset- zen normalen Stuhls an dafür nicht vorgesehenen Stellen (z.B. in die Kleidung oder auf den Boden) bei Kindern nach Abschluss des vierten Lebensjahrs (oder einer vergleichbaren Entwicklungsstufe) ohne organische Ursache [2], gilt Stuhlschmieren (Soiling) als der unwillkürliche Abgang kleiner Stuhlmengen, die Spuren in der Unterwäsche hinterlassen [1]. Weltweit gesehen verwenden Kinderärzte die Bezeichnung Enkopresis in verschiedenen Zusammenhängen. Während die Enkopresis von Kinderärzten in den USA häufig als Ausdruck einer gestörten Aggressionssteuerung gesehen wird, wird sie von Kinderärzten in Europa und Australien eher mit Obstipation assoziiert.

Die Stuhlinkontinenz ist das wichtigste Symptom der Obstipation im Kindesalter [3]. Die Stuhlinkontinenz ist ein allgemein bekanntes, von den Eltern leicht zu bewertendes Symptom. In unserer Ambulanz verwenden wir ferner die Häufigkeit der unwillkürlichen Stuhlentleerung und die Stuhlmenge in der Unterwäsche als objektive Marker für die Schwere der Obstipation und die Wirksamkeit der Behandlung. Es überrascht daher, dass die Stuhlinkontinenz in den 1999 im Rahmen der RomII-Kriterien aufgestellten Definitionen der funktionellen, d.h. der nichtorganischen, Obstipation und der funktionellen Stuhlretention nicht enthalten war [4]. Zwei jüngere Studien untersuchten die Anwendbarkeit der RomII-Kriterien in der klinischen Praxis $[3,5]$. Voskuijl et al. [3] bewerteten 130 Patienten, die unter Obstipation bzw. Stuhlinkontinenz litten und stellten fest, dass die Erkrankung bei Zugrundelegung der Rom-II-Kriterien bei 16\%

\section{KARGER \\ Fax +41613061234 \\ E-Mail karger@karger.ch}

www.karger.com (c) 2008 Nestec Ltd., Vevey/S. Karger AG, Basel

0250-9652/07/0652-0081\$23.50/0

Online-Zugriff auf:

www.karger.com/and
M.E.J. Bongers, MD

Department of Pediatric Gastroenterology and Nutrition, Room C2-312

Academic Medical Centre, Meibergdreef 9

NL-1105 AZ Amsterdam (The Netherlands)

Tel. +31 20566 2906, Fax +31 20566 9478, E-Mail mbongers@amc.nl 
Tabelle 1. Rom-III-Kriterien

1 Funktionelle Obstipation: Bei Kleinkindern im Alter von bis zu 4 Jahren müssen mindestens zwei der unten aufgeführten Kriterien über eine Dauer von mindestens einem Monat vorliegen:

Zwei oder weniger Stuhlentleerungen pro Woche

Nach Erlernen der Stuhlkontrolle mindestens eine Episode der Stuhlinkontinenz pro Woche

Übermässige Stuhlretention in der Anamnese

Schmerzhafter oder harter Stuhlgang in der Anamnese

Grosse Stuhlmassen im Rektum

Grosse Stuhlmassen, welche die Toilette verstopfen können, in der Anamnese

Keine erkennbaren strukturellen, endokrinen oder metabolischen Ursachen

2 Funktionelle nicht-retentive Enkopresis: Bei Kindern mit einem Entwicklungsalter von mindestens 4 Jahren müssen die folgenden Beschwerden vor der Diagnose mindestens zwei Monate lang vorgelegen haben:

Mehr als eine Episode der im sozialen Kontext

unpassenden Stuhlentleerung pro Monat

Keine erkennbaren entzündlichen, anatomischen, metabolischen oder neoplastischen Prozesse, welche die Symptome des Patienten erklären würden

Keine erkennbare Stuhlretention

der betroffenen Patienten nicht entdeckt wurde [3]. Loening-Baucke [5] untersuchte 213 Kinder mit Stuhlinkontinenz, um zu bewerten, ob diese Kinder die Rom-II-Kriterien für funktionelle Stuhlretention erfüllten. Obwohl in der Anamnese bzw. der klinische Untersuchung bei 85\% der Kinder Anzeichen einer Stuhlretention entdeckt wurden, erfüllten nur $41 \%$ der Kinder die Rom-II-Kriterien. Beide Studien kamen zu dem Ergebnis, dass die Rom-II-Kriterien zu restriktiv sind und viele der unter Obstipation leidenden Patienten nicht erfassen. Ausgehend von den in diesen Studien gemachten Empfehlungen wurde die Stuhlinkontinenz als ein Kriterium der funktionellen Obstipation in die Rom-III-Kriterien aufgenommen. Ferner wurden in den Rom-III-Kriterien die Begriffe Enkopresis und Stuhlschmieren durch den neutraleren Begriff funktionelle Stuhlinkontinenz ersetzt. Die funktionelle Stuhlinkontinenz lässt sich weiter unterteilen in die mit der funktionellen Obstipation (functional constipation, FC) zusammenhängende retentive Enkopresis und die funktionelle nicht-retentive Enkopresis (functional non-retentive fecal incontinence, FNRFI). Die Rom-III-Kriterien für die funktionelle Obstipation und die funktionelle nicht-retentive Enkopresis sind in Tabelle 1 zusammengefasst [6].

\section{Epidemiologie}

Laut einer systematischen Übersichtsarbeit jüngeren Datums beträgt die Prävalenz der Obstipation im Kindesalter weltweit in der allgemeinen Bevölkerung zwischen 0,7 und 29,6\% (Median 8,9; interquartiler Abstand 5,3-17,4) [7]. Die meisten Studien berichten über ähnliche Prävalenzraten bei Jungen und Mädchen [7]. Zum Thema Stuhlinkontinenz bei Kindern mit Obstipation wurden nur zwei epidemiologische Studien gefunden. Eine an 1975 griechischen Kindern im Alter zwischen 2 und 14 Jahren durchgeführte Studie ergab eine beträchtlich höhere Rate an Stuhlinkontinenz bei den unter Obstipation leidenden Kindern als bei den Kontrollkindern (25 vs. 0,4\%) [8]. Im Gegensatz dazu ergab eine an 319 brasilianischen Kindern im Alter zwischen 8 und 10 Jahren durchgeführte Studie keine Unterschiede bezüglich der Stuhlinkontinenz bei Kindern mit und Kindern ohne Obstipation (19 vs. 14\%) [9]. Für die niedrige Prävalenz der Stuhlinkontinenz bei den Kindern mit Obstipation und die hohe Prävalenz bei den Kindern ohne Obstipation wurde keine plausible Erklärung geliefert.

Zur Prävalenz der Stuhlinkontinenz gibt es nur wenige epidemiologische Studien und diese sind in der Regel veraltet. 1966 berichtete Bellmann [2], die Prävalenz der Stuhlinkontinenz betrage bei Kindern über 4 Jahren $1,3 \%$. In der Altersgruppe der 7- bis 8-Jährigen betrug die Prävalenz bei Jungen 2,3\% und bei Mädchen 1,3\% und in der Altersgruppe der 10- bis 12-Jährigen 1,3 bzw. 0,3\% [2, 10]. Die Stuhlinkontinenz trat häufiger beim männlichen Geschlecht auf, wobei das Verhältnis männlich zu weiblich zwischen 3:1 und 6:1 beträgt $[2,11,12]$. Eine jüngere populationsbasierte Studie zur Prävalenz der Stuhlinkontinenz erhob bei 13111 Kindern im Alter zwischen 5 und 6 Jahren und 9780 Kindern im Alter zwischen 11 und 12 Jahren die Daten zur Stuhlinkontinenz [13]. Die Gesamtprävalenz der Stuhlinkontinenz belief sich auf $3,0 \%$. Die Stuhlinkontinenz trat häufiger in der Altersgruppe der 5- bis 6-Jährigen als bei den 11- bis 12-Jährigen $(4,1$ vs. $1,6 \%)$ und häufiger bei Jungen als bei Mädchen auf (3,7 vs. $2,4 \%)$ [13].

Die Tatsache, dass in keiner der veröffentlichten epidemiologischen Studien zur Stuhlinkontinenz zwischen der funktionellen Obstipation und der funktionellen nicht-retentiven Enkopresis unterschieden wurde, ist eine eindeutige Unzulänglichkeit. Künftig sollten in grossen populationsbasierten Studien die Rom-III-Kriterien verwendet werden, um einen tieferen Einblick in die unterschiedliche Prävalenz dieser beiden Erkrankungen zu erhalten. 


\section{Pathophysiologie}

Die Obstipation im Kindesalter setzt für gewöhnlich in den ersten vier Lebensjahren ein. Die Stuhlverhaltung spielt bei der Entstehung bzw. Persistenz der Obstipation eine wichtige Rolle. Man geht davon aus, dass der Zeitpunkt der Sauberkeitserziehung eine kritische Phase ist, in der es infolge des zwischen Kind und Eltern ausgetragenen 'Kampfes' zur Obstipation kommen kann [14]. Borowitz et al. [15] fanden interessanterweise keinen $\mathrm{Zu}$ sammenhang zwischen dem Zeitpunkt, der Art und den Methoden der Sauberkeitserziehung einerseits und der Entstehung einer Obstipation im Kleinkindalter andererseits. Die Stuhlverhaltung bei Kindern kann auf diverse andere Ursachen zurückzuführen sein: (1) Erfahrungen mit schmerzhaften oder harten Stuhlgängen in der Vergangenheit; (2) Analfissuren; (3) zu wenig Zeit für eine regelmässige Stuhlentleerung, und (4) Widerstand seitens des Kindes, fremde Toiletten zu benutzen [15].

Je länger der Stuhlgang zurückgehalten wird, desto schwerer wird die Stuhlentleerung. Dies führt zu einem Teufelskreis, bei dem das Rektum durch abnorm feste und grosse Stuhlmengen zunehmend gedehnt wird [1]. Eine chronische Überdehnung des Rektums führt dann in der Folge zur Überlaufenkopresis. Eine chronische Stuhlimpaktion kann mit der Zeit zu einem dilatierten Rektum mit herabgesetztem Rektaltonus und reduzierter rektaler Kontraktilität führen, wodurch sich die Stuhlentleerung noch weiter verzögert [16]. Letztendlich führt dann möglicherweise eine gestörte Rektalfunktion und nicht die Stuhlverhaltung zu einer persistierenden obstruktiven Defäkationsstörung. Voskuijl et al. [17] berichteten bei Kindern mit Obstipation über eine höhere Dehnbarkeit des Rektums (rektale Compliance) als bei gesunden Kindern. Dieses Ergebnis deutet darauf hin, dass das Rektum gedehnt wird und daher grössere Stuhlmengen nötig sind, um eine rektale Empfindung, wie z.B. Stuhldrang auszulösen. Bei Kindern mit Obstipation und Kindern mit funktioneller nicht-retentiver Enkopresis [17] wurde keine Störung des rektalen Empfindens festgestellt. Zwar liegt bei den meisten Kindern mit chronischer Obstipation eine Störung der rektalen Compliance vor, aber die klinische Relevanz dieser Störung wurde bislang nicht untersucht. Bei Kindern mit funktioneller nicht-retentiver Enkopresis unterschied sich die rektale Compliance nicht von der rektalen Compliance gesunder Kinder - ein Ergebnis, das dafür spricht, dass es sich bei der funktionellen nicht-retentiven Enkopresis um eine eigenständige Erkrankung handelt $[17,18]$.

Funktionelle Stuhlinkontinenz bei Kindern
Die Stuhlinkontinenz wird häufig mit Verhaltensproblemen in Verbindung gebracht. Laut der inzwischen überholten Meinung der psychiatrischen Fachliteratur leiden Kinder mit Stuhlinkontinenz unter massiven Verhaltensstörungen, die durch eine passiv/aggressive Persönlichkeit gekennzeichnet sind [2, 19, 20]. Es wurde die These aufgestellt, diese Verhaltensprobleme seien die Ursache der Stuhlinkontinenz und die betroffenen Kinder müssten daher psychiatrisch behandelt werden [19]. Gabel et al. [21] fanden später heraus, dass Kinder mit Stuhlinkontinenz in der Tat häufiger unter Verhaltensstörungen leiden als Kinder aus der Kontrollgruppe, jedoch nicht in dem Ausmass, wie es bei Kindern, die an einen Dienst zur Versorgung psychischer Erkrankungen überwiesen werden, üblich ist. Zwei epidemiologische Studien jüngeren Datums stellten bei Kindern mit Stuhlinkontinenz jeweils höhere Raten an emotionalen Störungen und Verhaltensauffälligkeiten fest als bei Kindern ohne Stuhlinkontinenz $[13,22]$. Kinder, bei denen häufiger Episoden der Stuhlinkontinenz (mindestens einmal wöchentlich) auftreten, leiden öfter unter diesen Problemen als Kinder, bei denen die Stuhlinkontinenz nur gelegentlich (seltener als einmal wöchentlich) auftritt [22]. Diese Studien geben jedoch keine Antwort auf die Frage, ob die psychischen Probleme Ursache oder Folge der Stuhlinkontinenz sind.

Mit den Verhaltensproblemen von Kindern mit retentiver Enkopresis vor und nach der Behandlung haben sich mehrere Studien befasst. Levine et al. [23] stellte im Vergleich mit einer Kontrollgruppe fest, dass Kinder mit Stuhlinkontinenz vermehrt sozial zurückgezogen waren und affektive Veränderungen aufwiesen. Eine Besserung der Stuhlinkontinenz nach der Behandlung ging mit einer allgemeinen Verbesserung der Verhaltensprofile einher. Gleichermassen stellte Young [24] fest, dass die erfolgreiche Behandlung der Stuhlinkontinenz bei Kindern eine Abnahme der Verhaltensprobleme und eine Verbesserung der Sozialkompetenz zur Folge hatte.

Eine Studie bewertete die Verhaltensprofile von Kindern mit funktioneller nicht-retentiver Enkopresis [25]. Bei 35\% dieser Kinder wurden anfänglich abnorme Verhaltensscores beobachtet. Eine erfolgreiche Behandlung wurde mit einer Besserung der Verhaltensscores assoziiert. Diese drei Studien bekräftigen die These, dass die Stuhlinkontinenz an der Ätiologie von Verhaltensproblemen beteiligt ist und nicht primär als psychische Störung klassifiziert werden kann. Dies unterstreicht die Vermutung, dass die Erstbehandlung von Kindern mit Stuhlinkontinenz in einer pädiatrischen Klinik erfolgen kann.

Ann Nestlé [Ger] 2007;65:81-89 


\section{Klinische Präsentation}

Die Stuhlinkontinenz ist eine besonders peinliche und erschreckende Erkrankung mit beträchtlichen medizinischen, sozialen und wirtschaftlichen Auswirkungen. Bei den meisten Kindern genügen eine gründliche Anamnese und eine klinische Untersuchung zur Differenzialdiagnose zwischen der funktionellen Obstipation und der funktionellen nicht-retentiven Enkopresis. Die nachfolgenden Fallberichte beschreiben zwei Patienten unserer Ambulanz und illustrieren die wichtigsten klinischen Unterschiede zwischen diesen beiden Erkrankungen.

\section{Fallbericht 1}

Ein achtjähriges Mädchen mit Stuhlinkontinenz wurde von ihrem Kinderarzt in unsere ambulante Klinik überwiesen. Die Entleerung des Mekoniums erfolgte innerhalb von 24 Stunden nach der Geburt und bei der Patientin traten im Säuglings- und Kleinkindalter keine Probleme mit der Stuhlentleerung auf. Die Sauberkeitserziehung war im Alter von drei Jahren abgeschlossen. Bei Schuleintritt im Alter von vier Jahren stellte das Mädchen jedoch die Stuhlentleerung auf der Toilette ein. Als die Patientin in unserer ambulanten Klinik vorstellig wurde, kotete sie täglich, manchmal auch nachts, zwei- bis dreimal ein. Alle zwei Wochen kam es bei dem Mädchen zur Entleerung einer grossen Stuhlmenge, welche die Toilette verstopfte. Die Stuhlkonsistenz war hart. Das Mädchen klagte häufig über Bauchschmerzen und Appetitmangel, wobei diese Symptome eindeutig mit dem Defäkationsmuster zusammenhingen. Verspürte das Mädchen einen Stuhldrang, überkreuzte es die Beine, um den Stuhl zurückzuhalten. Eine Behandlung mit Lactulose ( $15 \mathrm{ml} / \mathrm{Tag}$ ) ergab keine Verbesserung. Die Untersuchung des Abdomens ergab keinen pathologischen Befund. Bei der Untersuchung des Rektums wurde jedoch perianal Stuhl festgestellt. Bei der digitalen Untersuchung des Rektums konnte eine grosse Stuhlmasse nachgewiesen werden.

\section{Fallbericht 2}

Ein Allgemeinarzt überwies einen achtjährigen Jungen mit Stuhlinkontinenz an unsere Ambulanz. Die Entleerung des Mekoniums erfolgte innerhalb von 48 Stunden nach der Geburt. Der Junge konnte im Alter von drei Jahren seinen Schliessmuskel tagsüber vollständig kontrollieren, kotete jedoch ab einem Alter von 5 Jahren häufig ein, wobei keine klare Ursache für das Einkoten festzustellen war. Trotz normaler Stuhlhäufigkeit wurde der Junge mit diversen Laxativa, u.a. Lactulose und Polyethylenglykol 3350, behandelt. Diese Behandlungsregimes mit Abführmitteln führten jedoch nicht zu einer Verringerung, sondern eher zu einer massiven Verstärkung der Stuhlinkontinenz. Nach Absetzen der Abführmittel kam es weiterhin einmal täglich zur Stuhlentleerung, wobei der Stuhl normal beschaffen war. Nichtsdestrotrotz stellte sich bezüglich der Stuhlinkontinenz keine Änderung ein. Im Gegensatz zu Kindern mit schwerer chronischer Obstipation, die mehrmals täglich einkoten, kam es bei unserem Patienten mit funktioneller nicht-retentiver Enkopresis stets einmal täglich und zwar nach der Schule, beim Spiel mit seinen Freunden zur Stuhlinkontinenz. Verspürte er einen Stuhldrang, dann verschob der Patient häufig den Gang zur Toilette. Sein Appetit war normal und er klagte nicht über Bauchschmerzen. Der Junge nässte ausserdem nachts ein. Die Untersuchung des Abdomens und des Rektums ergab keinen Hinweis auf Stuhlretention.

\section{Klinische Symptome}

Bei ungefähr 85\% der Kinder mit Obstipation kommt es infolge einer schweren Stuhlimpaktion zur Stuhlinkontinenz [3]. Entsprechend der klinischen Präsentation unserer ersten Patientin leiden Kinder mit funktioneller Obstipation (retentiver Enkopresis, bzw. Einkoten im Zusammenhang mit Stuhlverstopfung) unter einer Kombination der folgenden Symptome: zu seltene Stuhlentleerung, Entleerung grosser Stuhlmengen, harter Stuhlgang, Einkoten und verkrampfte Körperhaltung. Aufgrund der seltenen Defäkation kommt es bei vielen Patienten zur sporadischen Entleerung grosser Stuhlmengen. Der Entleerung grosser Stuhlmengen gehen oft Episoden mit vermehrtem Einkoten, Bauchschmerzen und Appetitlosigkeit voraus. Diese Symptome verschwinden unmittelbar nach der Stuhlentleerung [26]. Die Stuhlinkontinenz ist häufig die Folge einer Impaktion des Rektums, die tagsüber und in schweren Fällen auch nachts zur Überlaufenkopresis führt. Die digitale Untersuchung des Abdomens bzw. des Rektums zeigt bei ungefähr 40 bis $80 \%$ der Kinder mit Obstipation einen Kotballen (Scybalum) im Rektum, wie bei unserer Patientin beschrieben $[5,27]$. Die rektale Untersuchung bietet bei Kindern mit Stuhlinkontinenz wertvolle Informationen, da die Stuhlverhaltung auf das Rektum beschränkt sein kann und daher bei einer Untersuchung des Abdomens eventuell übersehen wird. Zwei Studien zeigten, dass bei 8-14\% der Kinder das Vorliegen einer Stuhlverhaltung, die zur Diagnose Obstipation führte, in der rektalen Untersuchung festgestellt wurde $[3,5]$.

Bei dem 8-jährigen Jungen mit Stuhlinkontinenz, der in unserer Klink vorstellig wurde, lagen im Gegensatz dazu keine Anzeichen einer Obstipation vor. Die folgenden Symptome sind charakteristisch für die funktionelle nicht-retentive Enkopresis und bestätigen die Diagnose: normale Defäkationshäufigkeit in Kombination mit normaler Stuhlkonsistenz, keine Entleerung grosser Stuhlmengen und kein Nachweis einer Stuhlimpaktion bei der klinischen Untersuchung. Es ist allgemein bekannt, dass bei Kindern mit funktioneller nicht-retentiver Enkopresis, wie z. B. hier bei unserem Patienten, die Häufigkeit des Einkotens unter Behandlung mit Laxativa zu- anstatt abnimmt. Abführmittel sind bei diesen Kin- 
dern kontraindiziert, da ihre Defäkationshäufigkeit und Stuhlkonsistenz bereits normal ist. Kinder mit nicht-retentiver Enkopresis koten nachts seltener ein (12\%) als Kinder mit retentiver Enkopresis (30\%) [28], nässen dagegen aber sowohl tagsüber als auch nachts häufiger ein $(40-45 \%)$ als letztere $(25-29 \%)[29,30]$.

\section{Diagnostische Aufarbeitung}

\section{Anamnese und klinische Untersuchung}

Eine sorgfältige Anamnese und eine vollständige klinische Untersuchung sind die Eckpfeiler der Diagnose von funktioneller Obstipation und funktioneller nichtretentiver Enkopresis. Bei der Anamneseerhebung sollten auch die Stuhlgewohnheiten des Kindes von der Geburt bis zum Zeitpunkt der Anamnese erfragt werden. Informationen über das Alter, in dem die Darmprobleme erstmals aufgetreten sind, die Häufigkeit der Stuhlentleerung, die Stuhlkonsistenz und -menge, Blutverlust aus dem Rektum, Schmerzen bei der Stuhlentleerung, Entleerung grosser Stuhlmengen und eine verkrampfte Körperhaltung bei der Stuhlentleerung sind von grosser Wichtigkeit. Erfragt werden müssen auch die Häufigkeit des (täglichen bzw. nächtlichen) Einkotens und die Situationen, in denen es zu Einkoten kommt (beim Spielen im Freien, beim Fernsehen oder am Computer). Begleitsymptome, wie z.B. Bauchschmerzen, Appetitlosigkeit und Einnässen, sind zu bewerten. Die Anamnese sollte auch die Ernährung des Kindes sowie frühere Behandlungsstrategien ermitteln. Allgemeine Informationen über Wachstum, Medikamenteneinnahme und neuromuskuläre Entwicklung sind einzuholen. Auskünfte über psychische oder Verhaltensprobleme und besondere Ereignisse im familiären Umfeld, wie z.B. die Geburt von Geschwistern, die Scheidung der Eltern, der Tod eines Familienmitglieds sowie sexueller Missbrauch sind ebenfalls von wesentlicher Bedeutung.

Bei allen Kindern mit Defäkationsstörungen sollte stets eine vollständige klinische und neurologische Untersuchung durchgeführt werden [26]. Die Untersuchung des Abdomens liefert wertvolle Hinweise bezüglich der Ansammlung von Gas oder Stuhl. Die Untersuchung der Perianalregion gibt Aufschluss über Anusposition, Stuhl in der Perianalregion, Rötung, Dermatitis, Ekzeme, Risse, Hämorrhoiden und Narben. Werden in der Untersuchung Analfissuren und Narben nachgewiesen, die nicht auf medizinische Ursachen zurückzuführen sind, so muss die Möglichkeit des sexuellen Missbrauchs in Betracht gezogen werden. Analfissuren und Narben finden

Funktionelle Stuhlinkontinenz bei Kindern sich sehr viel häufiger bei Kindern, die in der Vergangenheit anal sexuell missbraucht wurden [31, 32]. Die anorektale digitale Untersuchung bewertet das perianale Empfinden, den Analtonus, die Grösse des Rektums, die Menge und Konsistenz des im Rektum befindlichen Stuhls, die willkürliche Kontraktion und Erschlaffung des Analsphinkters sowie das Vorliegen eines normalen Anokutanreflexes.

\section{Röntgenaufnahme des Abdomens und}

Kolon-Transitzeit

In der klinischen Praxis wird bei Kindern mit Anzeichen einer Obstipation bzw. mit Stuhlinkontinenz häufig eine normale Röntgenaufnahme des Abdomens angefertigt, um die Stuhlmenge im Darm nachzuweisen. Die Daten bezüglich des Nutzens einer solchen Röntgenaufnahme zur Diagnose der Obstipation sind jedoch widersprüchlich. Eine unlängst durchgeführte, systematische Übersichtsarbeit bewertete die in kontrollierten Studien gewonnenen Belege hinsichtlich des Zusammenhangs zwischen einer auf einer Röntgenaufnahme des Abdomens nachgewiesenen Stuhlimpaktion und den klinischen Anzeichen und Symptomen bei Kindern [33]. Zu diesem Thema konnten nur zwei qualitativ hochwertige Studien ermittelt werden. Ausgehend von diesen Studien kam man zu dem Schluss, dass auf der Basis einer Röntgenaufnahme die Diagnose Obstipation genauso häufig bei Kindern mit wie bei Kindern ohne klinische Obstipation gestellt wird [33].

Man vermutet, dass der zusätzliche Einsatz radioopaker Marker zur Bewertung der Kolon-Transitzeit (CTT) wertvollere Informationen über die Darmperistaltik liefert als eine normale Abdomen-Röntgenaufnahme. Bei der Methode nach Bouchoucha nehmen die Patienten an sechs aufeinanderfolgenden Tagen täglich eine Kapsel mit 10 Markern ein und am 7. Tag wird dann eine Röntgenaufnahme zur Berechnung der Kolon-Transitzeit angefertigt [34]. Mit dieser Methode lassen sich sowohl die vollständige Darmpassage als auch die Passage in den einzelnen Darmabschnitten bewerten und so verschiedene Obstipationsformen unterscheiden: (1) normale Kolon-Transitzeit $=$ normale Passage durch alle Darmabschnitte; (2) Outlet-Obstruktion = verzögerte Passage im Bereich des Enddarms und des Afters; (3) Slow-Transit-Obstipation $=$ verzögerte Passage durch den gesamten Darm (kologene Obstipation) [26]. Bei ungefähr 50\% der Kinder mit Obstipation ist die Passage durch den Darm verzögert [35-37], und zwar meist im Bereich des Enddarms und des Afters [35]. Es stellte sich heraus, dass die Symptome der schweren Obstipation, die von den betrof- 
fenen Kindern bzw. ihren Eltern in einem Stuhltagebuch aufgezeichnet wurden, stark mit einer verlängerten Passagezeit durch den Darm korrelierten [35]. Ferner wurde belegt, dass die von den Betroffenen in einem Stuhltagebuch aufgezeichneten Symptome mit den tatsächlichen Stuhlgewohnheiten des Kindes übereinstimmen [38]. Dies bestätigt, dass eine Bewertung der Passagezeit durch den Darm bei einer angemessenen Bestandsaufnahme der klinischen Symptome überflüssig ist. Bei der Unterscheidung von Kindern mit funktioneller Obstipation und Kindern mit funktioneller nicht-retentiver Enkopresis dagegen hat sich der Marker-Test als nützlich erwiesen. Die Passagezeit durch den Darm ist bei neunzig Prozent der Kinder mit funktioneller nicht-retentiver Enkopresis normal [36]. Ergibt die klinische Untersuchung keine Stuhlimpaktion und ist die Passagezeit durch den Darm und die Häufigkeit der Stuhlentleerung normal, so bestätigt dies die Diagnose funktionelle nicht-retentive Enkopresis.

\section{Magnetresonanztomographie des Rückenmarks}

Werden in der klinischen Untersuchung neurologische Auffälligkeiten nachgewiesen, gilt es, einen spinalen Dysraphismus, z.B. ein intradurales Lipom, ein Lipom des Filum terminale, Dermalsinus und Tight filum terminale, als Ursache auszuschliessen [39]. Alarmierende Anzeichen für eine neurologische Auffälligkeit sind u.a. motorische und sensorische Störungen der unteren Extremitäten, abnorme Reflexe bzw. auffällige Empfindungen im Anorektalbereich und ein abnormer Anokutanreflex $[39,40]$. Werden diese Auffälligkeiten bei Kindern festgestellt, muss eine Magnetresonanztomographie des Rückenmarks durchgeführt werden. In einer vor Kurzem durchgeführten Studie ergab die Magnetresonanztomographie bei 9\% der unter intraktabler Obstipation leidenden Kinder eine Rückenmarkanomalie. Nachdem diese Anomalie durch einen chirurgischen Eingriff behoben wurde, legte sich die Obstipation bei $86 \%$ dieser Kinder [40]. Bei der Studie handelte es sich um eine retrospektive Studie an Patienten mit schwerer Obstipation, die nicht auf eine aggressive Therapie zur Darmentleerung ansprachen, was die Interpretation der Ergebnisse natürlich einschränkt. Um mehr Klarheit über mögliche ursächliche Rückenmarkanomalien zu gewinnen, müssen künftig prospektive Studien an Kindern mit Obstipation durchgeführt werden.

\section{Anorektale Manometrie und Barostat}

Zur Bewertung der anorektalen Funktion können diverse Methoden eingesetzt werden. Die anorektale Ma- nometrie misst den Druck im Anorektalbereich und dient zur Bewertung der Sphinkterfunktion und der Kontraktionsmuster. Bei Kindern mit Obstipation kann die anorektale Manometrie zur Untersuchung des rektoanalen Inhibitionsreflex eingesetzt werden. Der Nachweis des rektoanalen Inhibitionsreflex dient dem Ausschluss von Morbus Hirschsprung. In der klinischen Praxis können diese Messungen nur von erfahrenen Ärzten bei Säuglingen durchgeführt werden, da die Interpretation der Untersuchung durch das Schreien und die Bewegungen der Säuglinge erschwert wird. Ferner ist die rektale Saugbiopsie der genaueste Test zur Diagnose von Morbus Hirschsprung und daher der anorektalen Manometrie vorzuziehen [41].

Die Bewertung der Kontraktionsmuster mittels anorektaler Manometrie ergab bei ungefähr $50-60 \%$ der Kinder mit Obstipation und Stuhlinkontinenz eine auffällige Defäkationsdynamik, z.B. das Unvermögen, bei der Defäkation den externen Analsphinkter zu entspannen $[25,42,43]$. Zwischen der Normalisierung der Defäkationsdynamik durch Biofeedback-Training und einem positiven klinischen Outcome wurde jedoch kein $\mathrm{Zu}$ sammenhang festgestellt, was darauf schliessen lässt, dass die auffällige Defäkationsdynamik bei diesen Kindern kein wichtiges pathophysiologisches Merkmal ist $[25,42]$.

Mittels anorektaler Manometrie wurde in mehreren Studien belegt, dass die rektale Sensitivität bei einem beträchtlichen Teil der unter Obstipation und Stuhlinkontinenz leidenden Kinder gestört ist [44, 45]. Die Messung der viszeralen Empfindung mittels volumengesteuerter Dehnung, z.B. bei der Manometrie, wird u.a. durch die unterschiedliche Compliance und die Grösse der Hohlorgane beeinflusst [46]. Studien mit Barostat-Messungen haben belegt, dass das Problem bei Patienten mit funktioneller Obstipation eher durch eine erhöhte Compliance verursacht wird als durch eine Sensitivitätsstörung [17]. Die klinischen Auswirkungen dieses Ergebnisses müssen noch näher untersucht werden. Barostat-Messungen an Kindern mit Obstipation bleiben daher erfahrenen Experten vorbehalten. Bei Kindern mit funktioneller nichtretentiver Enkopresis ist sowohl die Compliance als auch die rektale Sensitivität normal.

Zusammenfassend kann festgestellt werden, dass eine Routineuntersuchung mittels anorektaler Manometrie oder Barostat-Messung bei Kindern mit Obstipation und Stuhlinkontinenz nicht indiziert ist.
86

Ann Nestlé [Ger] 2007;65:81-89
Bongers/Benninga 


\section{Behandlung}

Die Behandlung der funktionellen Obstipation beruht auf einer Kombination aus Aufklärung von Eltern und Kind, Verhaltensänderungen und einer Behandlung mit Abführmitteln [47]. Ein umfassender Überblick über die multimodale Therapie bei Obstipation im Kindesalter erfolgt an anderer Stelle in dieser Ausgabe der Annales Nestlé.

Abgeleitet von dem Behandlungsansatz für Kinder mit Obstipation, werden Kinder mit funktioneller nichtretentiver Enkopresis konventionell durch Aufklärung, Verhaltensänderung (mittels Toilettentraining dreimal täglich nach den Mahlzeiten) und dem Führen eines 'Stuhltagebuchs' behandelt [48]. Die Therapie muss sanft und frei von Schuldzuweisungen sein und ein Belohnungssystem hat sich daher als nützlich erwiesen. Abführmittel sind bei Kindern mit funktioneller nicht-retentiver Enkopresis kontraindiziert, da ihre Stuhlentleerungsfrequenz bereits normal ist [29]. Sonstige Optionen der medikamentösen Behandlung, z.B. mit Loperamid, wurden vorgeschlagen, bedürfen hinsichtlich ihrer Wirksamkeit zur Behandlung der funktionellen nicht-retentiven Enkopresis jedoch noch weiterer Untersuchungen [49]. Es gibt keinerlei Hinweis darauf, dass BiofeedbackTraining bei der Behandlung der funktionellen nicht-retentiven Enkopresis im Kindesalter eine zusätzliche Nutzwirkung zur konventionellen Therapie entfaltet [25]. Auch zur Rolle der psychologischen Therapie bei funktioneller nicht-retentiver Enkopresis stehen bisher keine Daten zur Verfügung. Die Frage, ob es zur Behandlung der funktionellen nicht-retentiven Enkopresis weiterer Therapiealternativen bedarf, muss künftig in randomisierten kontrollierten Studien mit wohl durchdachtem Studiendesign untersucht werden [25].

\section{Follow-up}

Kinder mit Obstipation wurden in diversen langfristigen Follow-up-Untersuchungen bewertet. Staiano et al. [50] beobachteten 62 Kinder über einen Zeitraum von 5 Jahren. Bei der Hälfte der Kinder mit Obstipation persistierten die Symptome während dieses Beobachtungszeitraums, d.h. bei diesen Kindern erfolgte die Stuhlentleerung auch weiterhin weniger als viermal wöchentlich. Interessanterweise sank die Häufigkeit der Stuhlinkontinenz unabhängig von dem bezüglich der Obstipation erzielten Outcome signifikant. In einer weiteren Studie mit einer mittleren Follow-up-Dauer von 4 Jahren wurden

Funktionelle Stuhlinkontinenz bei Kindern
66\% der 137 Kinder mit Obstipation geheilt, wobei der Begriff «Obstipation» in der Studie jedoch nicht eindeutig definiert war [51]. Das Vorliegen von Stuhlinkontinenz bei Einschluss in die Studie wurde nicht mit einem schlechteren Outcome während der Follow-up-Dauer assoziiert. Eine dritte, von Van Ginkel et al. durchgeführte Follow-up-Studie [52] bewertete 403 Kinder mit Obstipation. Eine erfolgreiche Behandlung wurde wie folgt definiert: Eine Stuhlentleerungsfrequenz von mindestens dreimal wöchentlich und weniger als zwei Stuhlinkontinenzepisoden über einen Zeitraum von vier Wochen. Insgesamt belief sich der Prozentsatz an erfolgreich behandelten Kindern während der gesamten Beobachtungsdauer von 8 Jahren auf $80 \%$, wobei bereits bei der nach einem Jahr durchgeführten Follow-up-Untersuchung eine Erfolgsrate von 60\% festgestellt worden war. Bei $50 \%$ der Kinder kam es nach anfänglichem Behandlungserfolg innerhalb der ersten 5 Jahre zu mindestens einem Rückfall. Bei einem Drittel aller Patienten persistierten die Symptome der Obstipation bis ins junge Erwachsenenalter. Im Gegensatz zu der zuvor erwähnten Studie wurde bei der Gruppe von Kindern, die bei Einschluss in die Studie nicht unter Stuhlinkontinenz litten, eine höhere Rate an positiven Outcomes erzielt [52].

Diese Ergebnisse belegen, dass die meisten Kinder, die unter Obstipation mit oder ohne Stuhlinkontinenz leiden, eine günstige Langzeitprognose haben. Andererseits jedoch widerlegen sie die allgemeine Annahme, die Obstipation vergehe bei den betroffenen Kindern mit Einsetzen der Pubertät von selbst. Die Symptome persistieren möglicherweise über das 18. Lebensjahr hinaus. Eine chronische Obstipation im Kindesalter wird mit einer verringerten Lebensqualität assoziiert [53]. Auch die Kinder selbst berichteten, in erster Linie aufgrund ihres beeinträchtigten physischen Leistungsvermögens, über eine niedrigere Lebensqualität. Die von den Eltern der Kinder angegebene Lebensqualität war sogar noch niedriger als die der betroffenen Kinder, wobei die Angaben wahrscheinlich von der Symptomdauer bei den Kindern und dem Auftreten ähnlicher Symptome bei weiteren Familienmitgliedern beeinflusst wurden [53]. Die Lebensqualität ist nicht nur bei Kindern mit Obstipation, sondern bei allen Kindern mit funktionellen Störungen der Stuhlentleerung, gemäss der Definition der Rom-II-Kriterien für funktionelle Erkrankungen des GI-Trakts, niedriger als bei gesunden Kontrollpersonen [54]. Die Ärzte müssen sich bewusst sein, wie stark sich diese Störungen auf das Kind auswirken und wie gross die Sorgen sind, die sich die Eltern während der Behandlung ihrer Kinder machen. 
Eine kleine Studie untersuchte vor Kurzem die Beziehung zwischen der funktionellen Obstipation im Kindesalter und anderen funktionellen Störungen des Gastrointestinaltrakts, wie z.B. Obstipation und Reizdarmsyndrom im Erwachsenenalter [55]. Das Auftreten einer Obstipation im Kindesalter schien einer der Prädiktoren für das Reizdarmsyndrom im Erwachsenenalter zu sein. Diese Studie beobachtete jedoch nur 20 Patienten bis ins Erwachsenenalter, d.h. das Outcome der Obstipation im Kindesalter sollte in prospektiven, an einer grösseren Stichprobe durchgeführten Follow-up-Studien näher untersucht werden. Die Follow-up-Untersuchung von Kindern mit Obstipation ist wichtig, da Rückfälle nach erfolgreicher Behandlung und die Persistenz der Symptome bis ins Erwachsenenalter alles andere als selten sind.

\section{Schlussfolgerungen}

Die funktionelle Stuhlinkontinenz bei Kindern ist im Allgemeinen eine Folge der funktionellen Obstipation. Entscheidend ist, dass bei Kindern mit Stuhlinkontinenz in der klinischen Praxis zwischen der funktionellen Obstipation (functional constipation, FC) und der funktionellen nicht-retentiven Enkopresis (functional non-retentive fecal incontinence, FNRFI) unterschieden werden muss, da diese zwei Krankheiten unterschiedliche Behandlungsstrategien erfordern. Die Unterscheidung dieser beiden Erkrankungen beruht hauptsächlich auf einer sorgfältigen Anamneseerhebung und klinischen Untersuchung. Um weitere Einblicke in die Prävalenz, die Pathophysiologie und die langfristigen Outcomes der funktionellen Störungen des Gastrointestinaltrakts im
Kindesalter zu gewinnen, müssen künftig Studien mit einem wohldurchdachten Studiendesign durchgeführt werden. Es bleibt zu hoffen, dass die vor Kurzem definierten Rom-III-Kriterien den internationalen Vergleich der Ergebnisse künftiger Studien und die internationale Zusammenarbeit auf diesem Bereich erleichtern werden.

\section{Hauptaussagen}

(1) Bei der funktionellen Stuhlinkontinenz im Kindesalter muss gemäss der Rom-III-Kriterien für funktionelle gastrointestinale Störungen im Kindesalter zwischen der retentiven und der nicht-retentiven Enkopresis unterschieden werden.

(2)In der klinischen Praxis wird mittels eingehender Anamneseerhebung und gründlicher klinischer Untersuchung sorgfältig zwischen der retentiven und der nicht-retentiven Enkopresis unterschieden.

(3) Die klinische Untersuchung sollte zur Bewertung der Stuhlimpaktion zumindest eine digitale rektale Untersuchung umfassen, vorausgesetzt die Kinder ängstigen sich nicht zu sehr vor dieser Untersuchung.

(4) Bei Kindern mit funktioneller nicht-retentiver Enkopresis sind Defäkationsmuster und die Dauer der Darmpassage normal und in der klinischen Untersuchung wird keine Stuhlimpaktion nachgewiesen.

(5)Die hohe Rückfallrate und die Persistenz der Obstipation bis ins Erwachsenenalter zeigen, wie wichtig Follow-up-Untersuchungen bei Kindern mit Obstipation sind, unabhängig davon, ob die Obstipation mit Stuhlinkontinenz einhergeht oder nicht.

\section{Literatur}

1 Di Lorenzo C, Benninga MA: Pathophysiology of pediatric fecal incontinence. Gastroenterology 2004;126(suppl):33-40.

2 Bellman M: Studies on encopresis. Acta Paediatr Scand 1966;Suppl 170:7-151.

-3 Voskuijl WP, Heijmans J, Heijmans HS, et al: Use of Rome II criteria in childhood defecation disorders: applicability in clinical and research practice. J Pediatr 2004;145:213217.

4 Rasquin-Weber A, Hyman PE, Cucchiara S, et al: Childhood functional gastrointestinal disorders. Gut 1999;45:60-68.
5 Loening-Baucke V: Functional fecal retention with encopresis in childhood. J Pediatr Gastroenterol Nutr 2004;38:79-84.

-6 Rasquin A, Di Lorenzo C, Forbes D, et al: Childhood functional gastrointestinal disorders: child/adolescent. Gastroenterology 2006;130:1527-1537.

7 Van den Berg MM, Benninga MA, Di Lorenzo C: Epidemiology of childhood constipation: a systematic review. Am J Gastroenterol 2006;101:2401-2409.

8 Roma-Giannikou E, Adamidis D, Gianniou $\mathrm{M}$, et al: Epidemiology of chronic constipation in Greek children. Hell J Gastroenterol 1999; 12:58-62.
-9 Araujo Sant'Anna AM, Calcado AC: Constipation in school-aged children at public schools in Rio de Janeiro, Brazil. J Pediatr Gastroenterol Nutr 1999;29:190-193.

10 Rutter M, Tizard J, Yule W, et al: Research report: Isle of Wight Studies, 1964-1974. Psychol Med 1976;6:313-332.

11 Levine MD: Children with encopresis: a descriptive analysis. Pediatrics 1975;56:412416.

12 Loening-Baucke V: Encopresis and soiling. Pediatr Clin North Am 1996;43:279-298. 
-13 van der Wal MF, Benninga MA, Hirasing RA: The prevalence of encopresis in a multicultural population. J Pediatr Gastroenterol Nutr 2005;40:345-348.

14 Huschka M: The child's response to coercive bowel training. Psychosom Med 1942;4:301308.

15 Borowitz SM, Cox DJ, Tam A, et al: Precipitants of constipation during early childhood. J Am Board Fam Pract 2003;16:213-218.

-16 Mertz H, Naliboff B, Mayer E: Physiology of refractory chronic constipation. Am J Gastroenterol 1999;94:609-615.

-17 Voskuijl WP, van Ginkel R, Benninga MA, et al: New insight into rectal function in pediatric defecation disorders: disturbed rectal compliance is an essential mechanism in pediatric constipation. J Pediatr 2006;148:6267.

$\checkmark 18$ Bongers ME, Tabbers MM, Benninga MA: Functional nonretentive fecal incontinence in children. J Pediatr Gastroenterol Nutr 2007;44:5-13.

-19 Bemporad JR, Kresch RA, Asnes R, Wilson A: Chronic neurotic encopresis as a paradigm of a multifactorial psychiatric disorder. J Nerv Ment Dis 1978;166:472-479.

20 Hoag JM, Norriss NG, Himeno ET, Jacobs J: The encopretic child and his family. J Am Acad Child Psychiatry 1971;10:242-256.

-21 Gabel S, Hegedus AM, Wald A, et al: Prevalence of behavior problems and mental health utilization among encopretic children: implications for behavioral pediatrics. J Dev Behav Pediatr 1986;7:293-297.

-22 Joinson C, Heron J, Butler U, Von Gontard A: Psychological differences between children with and without soiling problems. Pediatrics 2006;117:1575-1584.

-23 Levine MD, Mazonson P, Bakow H: Behavioral symptom substitution in children cured of encopresis. Am J Dis Child 1980;134:663667.

24 Young MH: Functional encopresis: symptom reduction and behavioral improvement. J Dev Behav Pediatr 1995;16:226-232.

-25 van der Plas RN, Benninga MA, Redekop WK, et al: Randomised trial of biofeedback training for encopresis. Arch Dis Child 1996; 75:367-374

-26 Benninga MA, Voskuijl WP, Taminiau JA: Childhood constipation: is there new light in the tunnel? J Pediatr Gastroenterol Nutr 2004;39:448-464.

-27 Hatch TF: Encopresis and constipation in children. Pediatr Clin North Am 1988;35: 257-280.
8 Benninga MA, Buller HA, Heymans HS, et al: Is encopresis always the result of constipation? Arch Dis Child 1994;71:186-193.

29 van Ginkel R, Benninga MA, Blommaart PJ, et al: Lack of benefit of laxatives as adjunctive therapy for functional nonretentive fecal soiling in children. J Pediatr 2000;137:808813.

30 Loening-Baucke V: Urinary incontinence and urinary tract infection and their resolution with treatment of chronic constipation of childhood. Pediatrics 1997;100:228-232.

31 Pierce AM: Anal fissures and anal scars in anal abuse - are they significant? Pediatr Surg Int 2004;20:334-338.

32 Bruni M: Anal findings in sexual abuse of children (a descriptive study). J Forensic Sci 2003;48:1343-1346.

33 Reuchlin-Vroklage LM, Bierma-Zeinstra S, Benninga MA, Berger MY: Diagnostic value of abdominal radiography in constipated children: a systematic review. Arch Pediatr Adolesc Med 2005;159:671-678.

34 Bouchoucha M, Devroede G, Arhan P, et al: What is the meaning of colorectal transit time measurement? Dis Colon Rectum 1992; 35:773-782.

35 de Lorijn F, van Wijk MP, Reitsma JB, et al: Prognosis of constipation: clinical factors and colonic transit time. Arch Dis Child 2004;89:723-727.

36 Benninga MA, Buller HA, Heymans HS, et al: Is encopresis always the result of constipation? Arch Dis Child 1994;71:186-193.

-37 Papadopoulou A, Clayden GS, Booth IW: The clinical value of solid marker transit studies in childhood constipation and soiling. Eur J Pediatr 1994;153:560-564.

-38 van der Plas RN, Benninga MA, Redekop $\mathrm{WK}$, et al: How accurate is the recall of bowel habits in children with defaecation disorders? Eur J Pediatr 1997;156:178-181.

39 Rossi A, Biancheri R, Cama A, et al: Imaging in spine and spinal cord malformations. Eur J Radiol 2004;50:177-200.

40 Rosen R, Buonomo C, Andrade R, Nurko S: Incidence of spinal cord lesions in patients with intractable constipation. J Pediatr 2004; 145:409-411.

41 de Lorijn F, Reitsma JB, Voskuijl WP, et al: Diagnosis of Hirschsprung's disease: a prospective, comparative accuracy study of common tests. J Pediatr 2005; 146:787-792.

42 van der Plas RN, Benninga MA, Buller HA, et al: Biofeedback training in treatment of childhood constipation: a randomised controlled study. Lancet 1996;348:776-780.
43 Sunic-Omejc M, Mihanovic M, Bilic A, et al: Efficiency of biofeedback therapy for chronic constipation in children. Coll Antropol 2002;26(suppl):93-101.

44 Molnar D, Taitz LS, Urwin OM, Wales JK: Anorectal manometry results in defecation disorders. Arch Dis Child 1983;58:257-261.

45 Meunier P, Marechal JM, de Beaujeu MJ: Rectoanal pressures and rectal sensitivity studies in chronic childhood constipation. Gastroenterology 1979;77:330-336.

46 Camilleri M: Testing the sensitivity hypothesis in practice: tools and methods, assumptions and pitfalls. Gut 2002;51(suppl 1):i34i40.

47 Constipation Guideline Committee of the North American Society for Pediatric Gastroenterology, Hepatology and Nutrition: Evaluation and treatment of constipation in infants and children: recommendations of the North American Society for Pediatric Gastroenterology, Hepatology and Nutrition. J Pediatr Gastroenterol Nutr 2006;43: e1-e13.

48 Voskuijl WP, Reitsma JB, van GR, et al: Longitudinal follow-up of children with functional nonretentive fecal incontinence. Clin Gastroenterol Hepatol 2006;4:67-72.

49 Voskuijl WP, van Ginkel R, Taminiau JA, et al: Loperamide suppositories in an adolescent with childhood-onset functional nonretentive fecal soiling. J Pediatr Gastroenterol Nutr 2003;37:198-200.

-50 Staiano A, Andreotti MR, Greco L, et al: Long-term follow-up of children with chronic idiopathic constipation. Dig Dis Sci 1994; 39:561-564.

-51 Keuzenkamp-Jansen CW, Fijnvandraat CJ, Kneepkens CM, Douwes AC: Diagnostic dilemmas and results of treatment for chronic constipation. Arch Dis Child 1996;75:3641.

52 van Ginkel R, Reitsma JB, Buller HA, et al: Childhood constipation: Longitudinal follow-up beyond puberty. Gastroenterology 2003;125:357-363.

53 Youssef NN, Langseder AL, Verga BJ, et al: Chronic childhood constipation is associated with impaired quality of life: a case-controlled study. J Pediatr Gastroenterol Nutr 2005;41:56-60.

54 Faleiros FT, Machado NC: Assessment of health-related quality of life in children with functional defecation disorders. J Pediatr (Rio J) 2006;82:421-425.

55 Khan S, Campo J, Bridge JA, et al: Long-term outcome of functional childhood constipation. Dig Dis Sci 2006;52:64-69. 